\title{
An experimental and theoretical investigation on the optical and photocatalytic properties of $\mathrm{ZnS}$ nanoparticles
}

\author{
F.A. La Porta ${ }^{\mathrm{a}, *}$, A.E. Nogueira ${ }^{\mathrm{b}}$, Lourdes Gracia ${ }^{\mathrm{c}}$, W.S. Pereira ${ }^{\mathrm{b}}$, G. Botelho ${ }^{\mathrm{b}}$, T.A. Mulinari ${ }^{\mathrm{d}}$, \\ Juan Andrés ${ }^{\mathrm{c}}$, E. Longo ${ }^{\mathrm{d}}$ \\ a Departamento de Química, Universidade Tecnológica Federal do Paraná, Londrina, Brazil \\ b Departamento de Química, Universidade Federal de São Carlos, São Carlos, Brazil \\ ${ }^{\mathrm{c}}$ Department of Analytical and Physical Chemistry, Univervisat Jaume I, Castelló de la Plana, Spain \\ d Instituto de Química, Universidade Estadual, Paulista, Araraquara, Brazil
}

\section{A R T I C L E I N F O}

\section{Keywords:}

$\mathrm{ZnS}$

Nanoparticles

Microwave-assisted solvothermal method

DFT

Photocatalysis

Photoluminescence

\begin{abstract}
A B S T R A C T
From the viewpoints of materials chemistry and physical chemistry, crystal structure directly determines the electronic structure and furthermore their optical and photocatalytic properties. Zinc sulfide (ZnS) nanoparticles (NPs) with tunable photoluminescence (PL) emission and high photocatalytic activity have been obtained by means of a microwave-assisted solvothermal (MAS) method using different precursors (i.e., zinc nitrate (ZN), zinc chloride (ZC), or zinc acetate (ZA)). The morphologies, optical properties, and electronic structures of the as-synthesized ZnS NPs were characterized by X-ray photoelectron spectroscopy (XPS), transmission electron microscopy (TEM), energy dispersive X-ray spectroscopy (EDX), Brunauer-Emmett-Teller (BET) isotherms for $\mathrm{N}_{2}$ adsorption/desorption processes, diffuse reflectance spectroscopy (DRS), PL measurements and theoretical calculations. Density functional theory calculations were used to determine the geometries and electronic

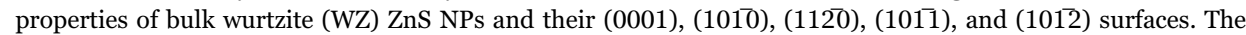
dependence of the PL emission behavior of ZnS NPs on the precursor was elucidated by examining the energy band structure and density of states. The method for degradation of Rhodamine B (RhB) was used as a probe reaction to investigate the photocatalytic activity of the as-Synthesised ZnS NPs under UV light irradiation. The PL behavior as well as photocatalytic activities of ZnS NPs were attributed to specific features of the structural and electronic structures. Increased photocatalytic degradation was observed for samples synthesized using different precursors in the following order: $\mathrm{ZA}<\mathrm{ZC}<\mathrm{ZN}$. These results indicated that samples synthesized with $\mathrm{ZN}$ present a greater percentage of exposed (0001) surface than those synthesized with the $\mathrm{ZC}$ and $\mathrm{ZA}$. Furthermore, the possible photodegradation mechanism of the as-prepared ZnS NPs were also briefly discussed.
\end{abstract}

\section{Introduction}

The interest in ZnS NPs has increase mainly because of their unique properties and enormous potential in emerging technologies ranging from optoelectronic luminescent devices to photocatalysis [1-8]. It is known that bulk $\mathrm{ZnS}$ is an important binary semiconductor with a direct, wide bandgap $(\sim 3.6 \mathrm{eV})$, high refraction index, and high transmittance in the visible range $[9,10]$. A variety of synthetic strategies has been used to obtain $\mathrm{ZnS}$ crystals with a superior control of their physical characteristics (e.g., sizes, well-defined morphologies, and compositions), including hot injection [11,12], solid state reaction [13], thermal decomposition [14], co-precipitation [15,16], hydro/ solvothermal method [13,17-21] and son on. On the other hand, the doping with impurities in ZnS NPs can display an increased efficiency in heterogeneous catalysis process, as well as in its optical properties [22-24]. However, despite recent advances in the chemical methods of synthesis, it is mandatory to conduct improvements with respect the existing synthetic methods in order to develop more efficient and environmentally friendly methods that allow the ability to synthesize these new complex materials with various functionalities and desired properties, as basis for the future development of new technologies at the nanoscale. In this context, more recently, our research group has employed the microwave-assisted solvothermal (MAS) method as a promising strategy to obtain $\mathrm{ZnS}$ crystals at shortened reaction times, low-temperatures and, ultimately, cleanness reactions compared to conventional routes $[4,9,21,25]$. Hence, there is one large interest in the development of the novel NPs based on $\mathrm{ZnS}$ with its tailored surfaces exposed to a variety of technological applications at the

\footnotetext{
* Corresponding author

E-mail addresses: felipe_laporta@yahoo.com.br, felipelaporta@utfper.edu.br (F.A. La Porta).
} 
nanoscale [4,22-29].

Intrinsic and visible emissions in $\mathrm{ZnS}$ NPs have been well-reported [30-32], and their PL properties have been explored by many researchers [9,25,33-36], while the photocatalytic properties of $\mathrm{ZnS}$ NPs have been analyzed by many authors for a variety of reactions [2729,37-42], however, the photocatalytic and PL behavior at nanoscale sizes remain far from well-understood.

First-principles calculations, mainly within the DFT framework, have proved invaluable in providing guidance and an atomistic understanding of the physical and chemical properties of emerging complex functional nanomaterials. Within this general framework, we are engaged in a joint experimental and theoretical project that is concerned with the synthesis, characterization, and technological applications of a series of $\mathrm{Pb}$ and Ag-based metal oxides, such as $\mathrm{PbMoO}_{4}$ [43], $\alpha-\mathrm{Ag}_{2} \mathrm{WO}_{4}$ [44], $\beta-\mathrm{Ag}_{2} \mathrm{WO}_{4}$ [45], $\beta-\mathrm{Ag}_{2} \mathrm{MoO}_{4}$ [46], $\beta-$ $\mathrm{AgVO}_{3}$ [47], $\mathrm{Ag}_{2} \mathrm{CrO}_{4}$ [48] or Ag-containing salts such as $\mathrm{Ag}_{3} \mathrm{PO}_{4}$ $[49,50]$.

Electronic and structural properties of the ZnS NPs as well as their surfaces can be revealed straightforwardly by means of theoretical calculations and have been studied extensively in the literature [5157]. For instance, Barnard et al. [52] reported the theoretical equilibrium morphologies of the ZnS NPs in the wurtzite (WZ) phase as a function of size, as determined by DFT calculations. Qin et al. [53] reported the photocatalytic efficiency of the zinc blend and WZ phases using first-principles DFT calculations for both the bulk samples and at the polar surfaces. Furthermore, the effect of water on crystallinity enhancement as well as on the structure transformation of ZnS NPs at room temperature has been reported in other theoretical studies [5557]. More recently, we reported the growth mechanism of ZnS NPs [4], and a DFT study of the structural and electronic properties of $\mathrm{ZnS}$ polymorphs and their pressure-induced phase transitions [51].

In this paper, a systematic investigation, based on the joint use of experimental data and theoretical calculations, into the morphologies, electronic structures, optical properties and photocatalytic activities of ZnS NPs has been presented. Three different precursors (i.e., ZN, ZC, or ZA) have been employed along the synthesis performed by means of the MAS method. These ZnS NPs were analyzed by XPS, TEM, EDX, BET isotherms for $\mathrm{N}_{2}$ adsorption/desorption processes, DRS, and PL measurements. Photocatalytic activities were evaluated via degradation of RhB under UV irradiation. DFT calculations allows us to carry out a detailed analyses of the electronic structures of both bulk WZ ZnS NPs

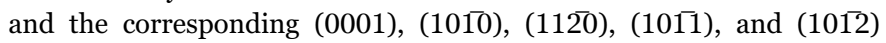
surfaces of the WZ ZnS and the origins of the intense PL bands and photocatalytic activities of $\mathrm{ZnS}$ were elucidated.

The rest of the paper is divided into three sections. Next, we present the experimental and theoretical methods and, our results presented are discussed in the section following the next. Main conclusions are summarized in the last section.

\section{Experimental and theoretical methods}

All reagents were analytical grade and used without further purification. ZnS NPs were prepared using the MAS method at $140{ }^{\circ} \mathrm{C}$ for $10 \mathrm{~min}$ using three different zinc salt precursors, i.e, $\mathrm{ZN}$, $\mathrm{ZC}$, or $\mathrm{ZA}$, in accordance with a protocol developed in a previous study [9]. Briefly, the synthesis of $\mathrm{ZnS}$ is described as follows: initially, $7.34 \mathrm{mmol}$ of precursors (i.e., ZN, ZA or ZC) and $15.44 \mathrm{mmol}$ of tetrabutylammonium hydroxide were dissolved in $25 \mathrm{~mL}$ of ethylene glycol (EG) and heated to $80{ }^{\circ} \mathrm{C}$ (solution 1). Afterward, $7.34 \mathrm{mmol}$ of thiourea were separately dissolved in another $25 \mathrm{~mL}$ of EG (solution 2. After some minutes, under vigorous magnetic stirring, the solution 2 was then quickly mixed in the solution 1 . Then, the mixture was transferred into a Teflon autoclave which was sealed and placed inside a domestic microwave-solvothermal system $(2.45 \mathrm{GHz}$, maximum power of $800 \mathrm{~W}$ ) at $140{ }^{\circ} \mathrm{C}$ for $10 \mathrm{~min}$. The resulting white solid was washed with deionized water and ethanol to remove byproducts possibly remaining in the final product and the precipitates were finally collected and dried at $70^{\circ} \mathrm{C}$ overnight. Based on this protocol, the XRD results confirm that all diffraction peaks can be indexed to the hexagonal structure of $\mathrm{ZnS}$ and the samples as-prepared have a high purity and crystallinity [9].

The obtained powders were structurally characterized using XPS spectra were collected using a commercial spectrometer (UNISPECSUHV) using the Mg Ka line ( $\mathrm{hv}=1253.6 \mathrm{eV})$. The composition of the surface region was determined from the ratio of the relative peak areas corrected by the sensitivity factors (Scofield) of the corresponding elements. Spectra were fitted without placing constraints using multiple Voigt profiles, and the BEs were corrected using the hydrocarbon component of adventitious carbon fixed at $285.0 \mathrm{eV}$. The morphologies were investigated using TEM images, which were recorded on a JEOL 2100 transmission electron microscope operated at $200 \mathrm{kV}$. During sample preparation for the TEM technique, the suspensions were deposited onto copper grids via fast immersion. BET isotherms for $\mathrm{N}_{2}$ adsorption/desorption processes were obtained by using a Micromeritics ASAP 2010 surface area and porosity analyzer.

The DRS spectra in the ultraviolet-visible region were recorded at room temperature between 200 and $800 \mathrm{~nm}$ using a Varian model Cary $5 \mathrm{G}$ in the diffuse reflectance mode. PL spectra were collected using a Thermal Jarrel-Ash Monospec monochromator and Hamamatsu R446 photomultiplier. The $350.7 \mathrm{~nm}(2.57 \mathrm{eV})$ exciting wavelength of a krypton ion laser (Coherent Innova) was used with the output of the laser maintained at $200 \mathrm{~mW}$. All measurements were performed at room temperature.

Photocatalytic activity of ZnS NPs synthesized with different zinc salts precursors was tested in the degradation of $\mathrm{RhB}$ under $\mathrm{UV}$. $\mathrm{RhB}$ is used as a model compound for oxidation reactions and has a strong absorption in the visible region $\left(\lambda_{\max }=554 \mathrm{~nm}\right)$. RhB is highly soluble in water and has properties similar to those of textile dyes, which are difficult to degrade. $\mathrm{RhB}$ dye solution $\left(80 \mathrm{~mL}, 10 \mathrm{mgL}^{-1}\right)$ at $\mathrm{pH} 6.0$ was mixed with $60 \mathrm{mg}$ of the catalyst and irradiated with an ultraviolet light placed in a box, which was set at a distance of $20 \mathrm{~cm}$ from the beaker containing the suspension. These systems remained under constant stirring in the dark for a period of $15 \mathrm{~min}$ to achieve adsorptiondesorption equilibrium on the surface of the ZnS NPs. After this time, the first aliquot was collected and considered as time zero. Photocatalytic tests were performed at $20^{\circ} \mathrm{C}$ in thermostatic bath with vigorous stirring. The UV radiation used in this experiment was obtained from a medium-pressure mercury vapor lamp (Osram, HQL 400) using the output without bulb protection. Reactions were monitored by UV-vis spectroscopy (JASCO V-660) at $554 \mathrm{~nm}$ using a commercial quartz cuvette.

To complement the experimental data, first-principles calculations were performed within the periodic DFT framework using the VASP program [58,59]. The Kohn-Sham equations were solved using the Perdew, Burke, and Ernzerh exchange-correlation functional [60], and the electron-ion interaction was described by the projector-augmentedwave pseudopotentials $[58,61]$. The plane-wave expansion was truncated at a cut-off energy of $520 \mathrm{eV}$ and is required for different convergence precisions, and the Brillouin zones were sampled through Monkhorst-Pack special k-points [62] grids $(6 \times 6 \times 1)$ that ensure geometrical and energetic convergence for the $\mathrm{ZnS}$ structures considered in this work. A vacuum spacer of $15 \AA$ was introduced in the zdirection to prevent the surfaces from interacting with each other. The polar (0001) and semi-polar (1011) surfaces were modeled with the top $\mathrm{Zn}$ layer and bottom $\mathrm{S}$ layer (each $\mathrm{S}$ atom with one dangling bond) using a $2 \times 2 \times 1$ conventional supercell with 20 at. layers. The (1010), $(1120)$ and $(10 \overline{2})$ facets were modeled by slabs containing twelve $\mathrm{ZnS}$ layers.

\section{Results and discussion}

High-resolution XPS spectra of the Zn 2p and S 2p orbitals for the 
(a)

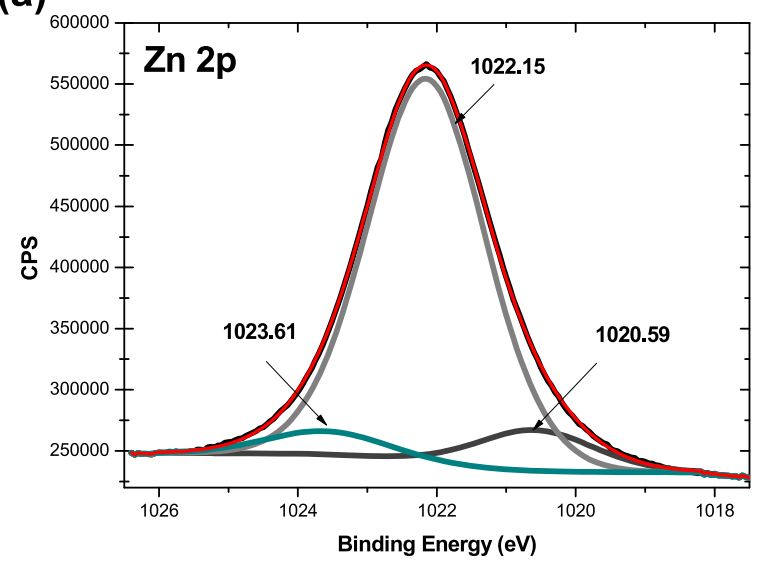

(c)

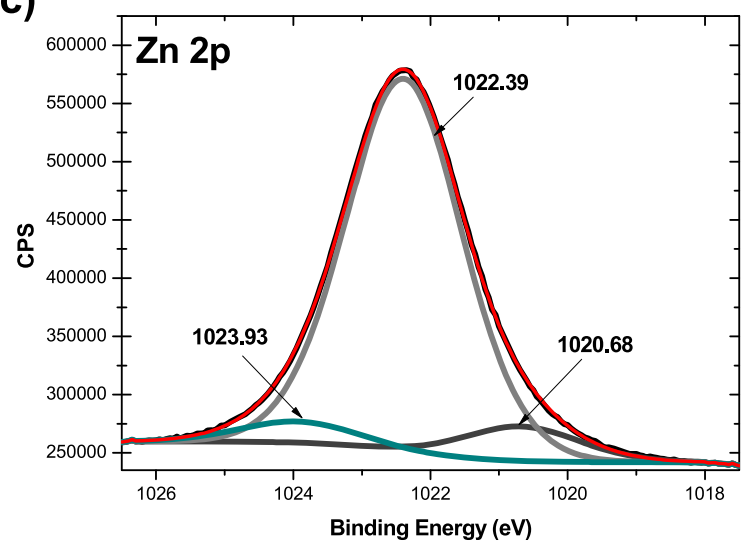

(e)

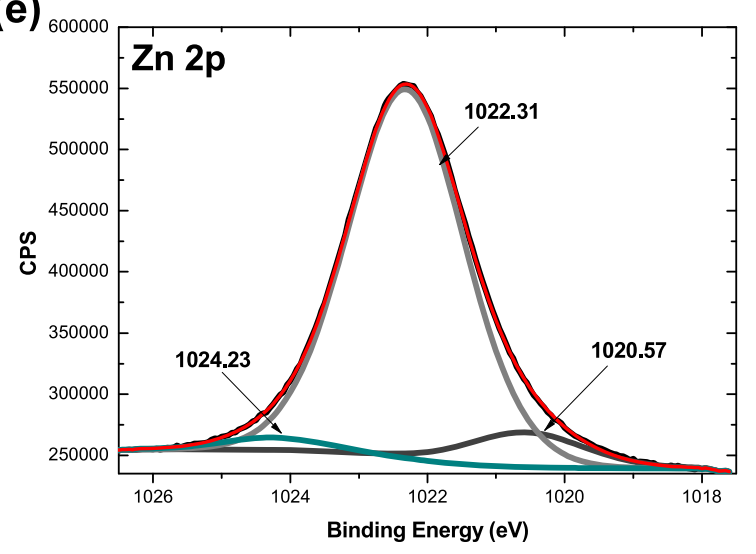

(b)

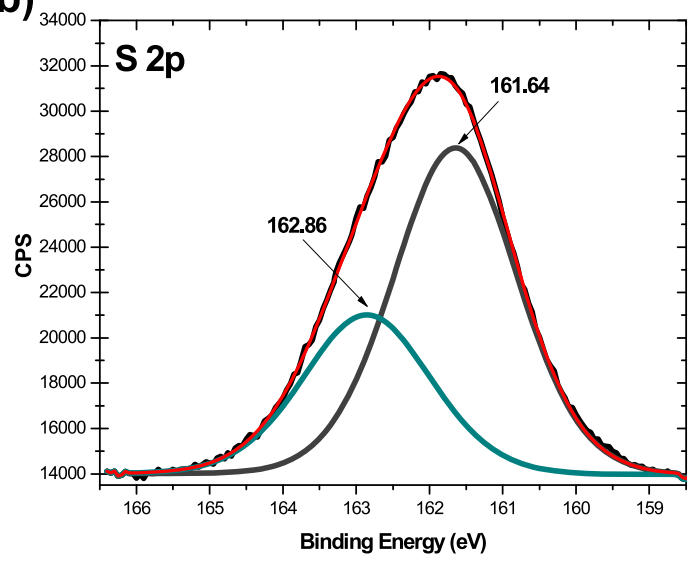

(d)

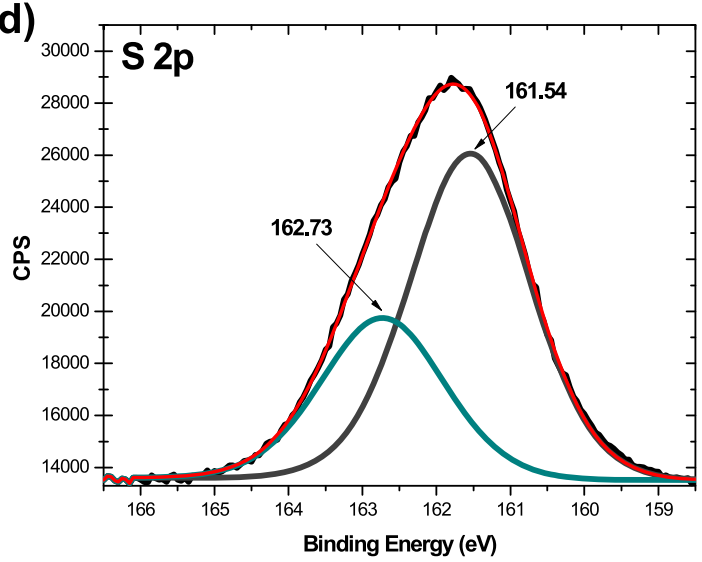

(f)

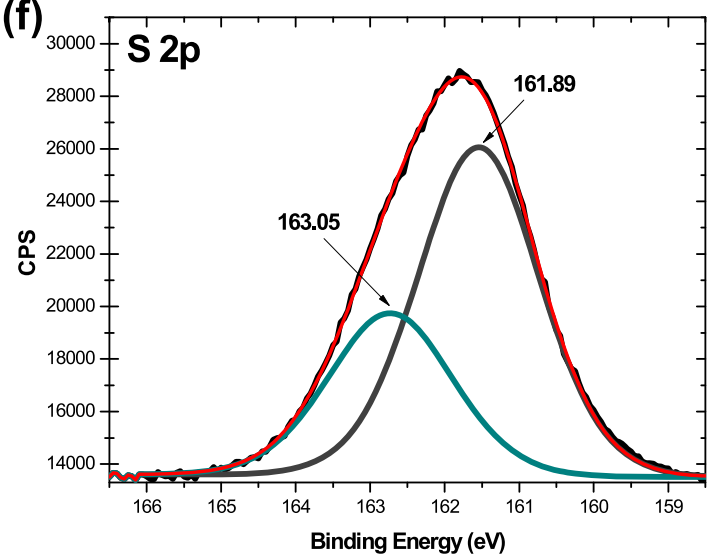

Fig. 1. : Zinc 2p3 and sulfur 2p XPS spectral of wurtzite ZnS NPs: (a-b) ZN; (c-d) ZC and (e-f) ZA.

WZ ZnS NPs are presented in Fig. 1. The high-resolution Zn 2p spectrum shows BEs around 1020,1022, and $1023 \mathrm{eV}$, and these values can be associated, respectively, with the presence of $\mathrm{Zn}-\mathrm{C}, \mathrm{Zn}-$ $\mathrm{S}$, and $\mathrm{Zn}-\mathrm{O}$ bonds at the surface, which is in accordance with other studies [9]. Moreover, it is important to remark that XPS results reveals a small amount of $\mathrm{Zn}-\mathrm{C}$ and $\mathrm{Zn}-\mathrm{O}$ bonds with respect $\mathrm{Zn}-\mathrm{S}$ bonds present in the XPS spectrum of all samples, which can be attributed to an insignificant contamination by such particles, i.e., hence the ZnS NPs prepared by the MAS method presents a wellcleaned surface and confirmed a high purity for such particles. The fitted S 2p spectra peaks (see Fig. 1) at approximately 161 and $163 \mathrm{eV}$ were assigned to $\mathrm{ZnS} 2 \mathrm{p}_{3 / 2}$ and $\mathrm{ZnS} 2 \mathrm{p}_{1 / 2}$ orbitals, respectively $[9,63,64]$. No signs of a secondary phase were observed using XPS spectra (this result is confirmed by TEM analysis, see supplementary material) for the ZnS NPs synthesized using different precursors. These results are consistent with $\mathrm{BE}$ values reported in the literature [63-65], and confirm the purity of ZnS NPs prepared using the MAS method.

On the other hand, the variations observed in the BEs are associated with the concentrations of sulfur defects on the surfaces of the ZnS NPs. Three different charge states of sulfur vacancies are evident: $\mathrm{V}_{\mathrm{S}}{ }^{\mathrm{x}}, \mathrm{V}_{\mathrm{S}}{ }^{*}$, and $\mathrm{V}_{\mathrm{S}}{ }$; these play a key role in understanding the physical and chemical properties of these NPs [9,63-67]. In addition, surface composition analysis via XPS measurements revealed ratios of $\mathrm{Zn} / \mathrm{S}$ atoms of 1:1, 1:0.9, and 1:0.8 for the ZnS NPs synthesized from $\mathrm{ZN}, \mathrm{ZC}$, or ZA, respectively.

The adsorption and desorption isotherm curves of the ZnS NPs generated from the three precursors are shown in the Supplementary Information. The specific surface areas were 233, 283, and $295 \mathrm{~m}^{2} \mathrm{~g}^{-1}$ for the ZnS NPs produced from ZN, ZC, or ZA precursors, respectively. The results showed Type I isotherms, which indicates the predomi- 
(a)

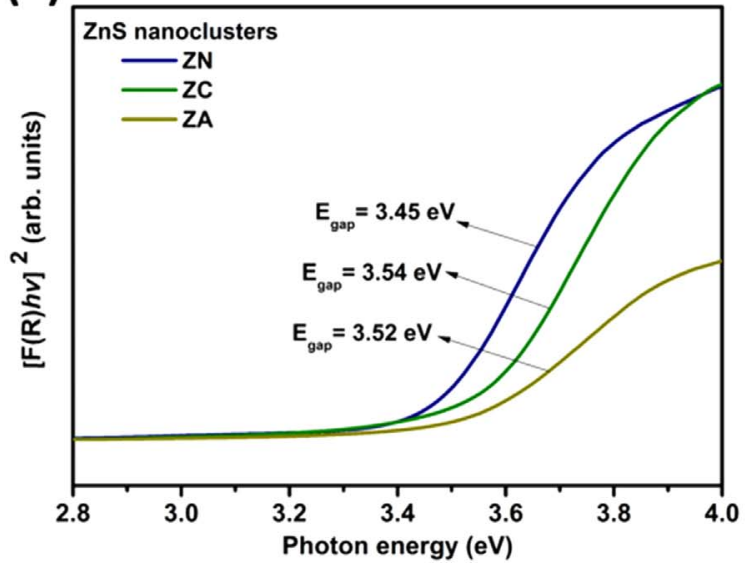

(b)

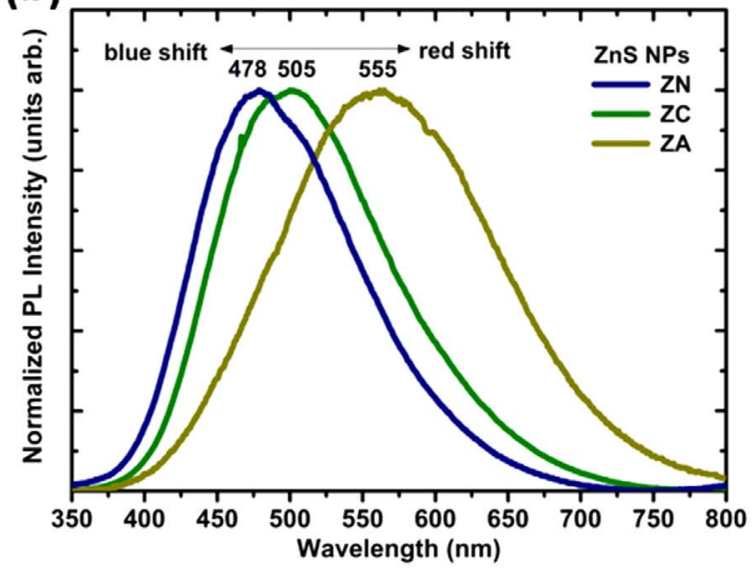

Fig. 2. : (a) DRS spectra and (b) PL spectra at room temperature of aggregated crystalline ZnS NPs obtained by a MAS method.

nance of mesopores in the three synthesized samples. These results are in good agreement with literature values [68]. Owing to the high surface areas of the ZnS NPs prepared using the MAS method, it is likely that these materials formed from small particles or clusters rather than single-crystalline $\mathrm{ZnS}$, which is in good agreement with our TEM results (see Supporting Information). Furthermore, the aggregation state for such NPs reveal a strong polarization in the ZnS surfaces and can in principle provide a rougher surface.

The optical properties were analyzed using DRS spectroscopy and PL measurements (see Fig. 2(a) and (b)). In particular, the DRS spectra in Fig. 2(a) shows variation in the optical direct band gaps of the $\mathrm{ZnS}$ NPs from 3.45 to $3.54 \mathrm{eV}$. For details on the calculations of the optical band gap see reference [9]. The band gap values estimated for the samples prepared using different precursors along the MAS synthesis are much lower than that expected for the band gap values of $3.77 \mathrm{eV}$ at room temperature for bulk WZ ZnS reported by Fang et al. [2]. Along the text, the term "defect" it is commonly used here in its broadest sense to encompass a disturbance in the ideal crystal framework, e.g., this definition may include dislocations, vacancies, presence of the impurities, even the surface of the material, and so on [69-71].

The variation in the DRS spectra is controlled by the degree of structural and electronic disorder on the crystalline phase of the $\mathrm{ZnS}$ NPs. This fact reveals that this disordered structure promotes local polarization and a charge gradient in the structure, which is consistent with the XPS results. Therefore, we believe that the structural changes provoked by the use of the different types of precursors can cause a variation in the optical band gap of the ZnS NPs, and hence is responsible for modulation of its physical and chemical properties, i.e., leading to different PL and photocatalytic behaviors of such NPs.

Fig. 2(b) shows the PL spectra at room temperature of ZnS NPs synthesized using the MAS process with different zinc salts. The PL spectra feature broad bands covering the visible electromagnetic spectrum from 400 to $800 \mathrm{~nm}$, with maximum emissions at 478, 505 , and $555 \mathrm{~nm}$ for the ZnS NPs synthesized using ZN, ZC, or ZA precursors, respectively, when excited by a $350.7 \mathrm{~nm}$ laser line. An analysis of the results points out the presence of red-shift of $0.36 \mathrm{eV}$, due to the structural changes in the ZnS NPs. This behavior, in principle, offers an excellent platform for the design and control of ZnS NPs that emit different colours via band-gap engineering.

An analysis of the PL emissions renders certain localized states that must exist in the forbidden band gap [4,9]. It has been reported that the PL emission of $\mathrm{ZnS}$ NPs in the visible region is due to sulfur vacancies $\left(\mathrm{V}_{\mathrm{S}}^{\mathrm{x}}, \mathrm{V}_{\mathrm{S}} \cdot\right.$, and $\left.\mathrm{V}_{\mathrm{S}}{ }^{* *}\right)$ that generate shallow and deep levels within the band gap $[9,24,25]$. Thus, a blue-shift in PL emission correlates with a higher concentration of shallow defect states while a red-shift corresponds to a greater concentration of deep trap states in these materials prepared using different zinc salts.

The PL behavior and catalytic activity of the ZnS NPs can be analyzed via the corresponding electronic structures. It is well-known that, after photoexcitation, the lattice configuration changes and distorted clusters appear in semiconductor materials allowing electrons to become trapped [69].

In this case, the high-energy photons can excite defect states of electrons localized at different energy levels within the band gap of the material. Therefore, structural defects in the bulk, surface, and/or interface may be trap sites, and electron-hole recombination frequently occurs at these trap sites. The higher surface-to-volume ratios of the ZnS NPs lead to a corresponding enhancement of surface-related emission in the overall luminescence [72,73].

There is a common perception that the predominant structural and electronic disorders in ZnS NPs are sulfur vacancies; it is considered that these are strongly dependent on the morphology, size, structure, and surface composition of the semiconductor materials and significantly affect the physical and chemical behavior of these systems [4]. The ratio of the concentrations of shallow and deep defects observed in the PL measurements can be estimated indirectly. The relationship between shallow and deep defects, $\rho$, is an important parameter for understanding the physical and chemical properties of semiconductor materials and can be estimated from the ratio of the left and right areas of the PL emission maximum. Thus, the relationship between shallow and deep defects can be defined as follows:

$\rho=[$ shallowdefects $] /[$ deepdefects $]$

Table 1 shows the estimated $\rho$ values obtained for the $\mathrm{ZnS}$ samples prepared using the MAS method. From this model, an increase in the $\rho$ value is related to a higher concentration of shallow defects, while a decrease in its value corresponds to a higher concentration of deep defects within the band gap. These results reveal that crystalline parameters may be obtained from the detailed analysis of the PL behavior, and hence can be used to build a close relationship between structure and properties of the ZnS NPs. This finding is very interesting for the monitoring of structural changes as well as their potential applications at the nanoscale. Hence, in this direction, the PL emission behavior may enable the development and the discovery of the new

Table 1

Values of $\rho$ for the ZnS NPs.

\begin{tabular}{llll}
\hline Sample & Area 1 (\%) & Area 2 (\%) & ( \\
\hline ZN & 86.41 & 53.78 & 1.61 \\
ZC & 85.72 & 66.30 & 1.29 \\
ZA & 109.57 & 86.28 & 1.27 \\
\hline
\end{tabular}



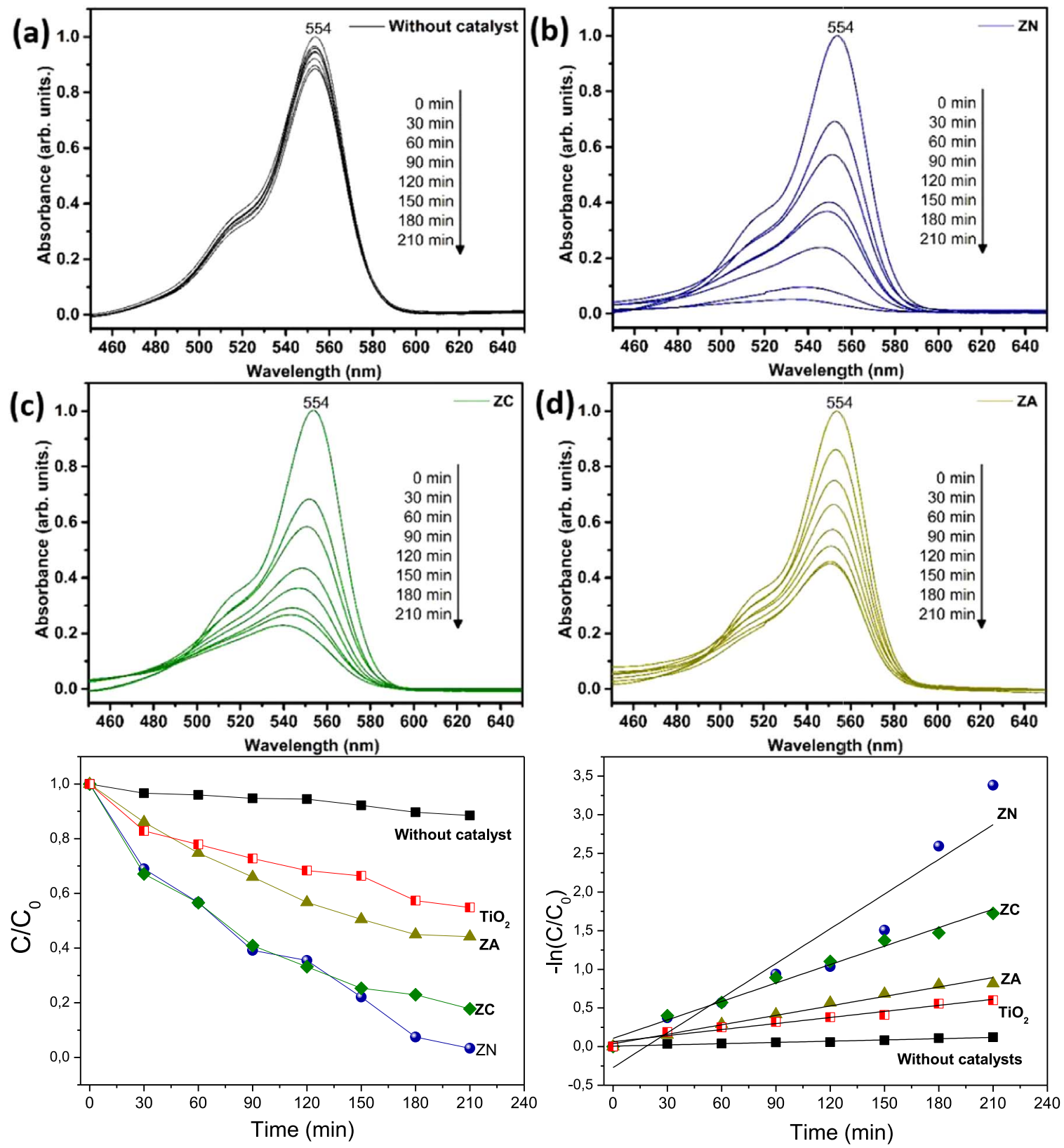

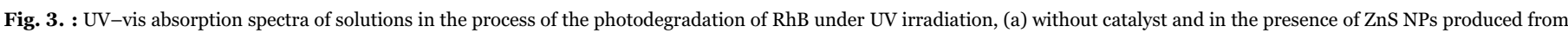
(b) ZN, (c) ZC and (d) ZA as precursors, respectively. (e) Photodegradation curves and (f) First-order kinetic curves for the discoloration of RhB solutions.

catalysts. Furthermore, the slope observed in the DRS spectra and blue-shift of the PL emission for the $\mathrm{ZnS}$ samples indicate varied crystallization of the samples synthesized using different precursors. Obviously, these zinc salts present different bonding forces and the crystalline structure strongly depends on the precursor used, which acts as a surface modifier causing different structural polarization effects on these NPs at short-, medium- and long-ranges, which can be extended to other complex systems. Based on these results, the binding strength of these precursors decreases as follows: $\mathrm{ZN}<\mathrm{ZC}<\mathrm{ZA}$. This fact provides an excellent opportunity for structural control of these complex functional materials and allows highlighting the crucial role of the precursor as a surface modifier. To further elucidate the structural and electronic properties of these NPs, theoretical calculations were performed.

The photocatalytic activities of the WZ ZnS NPs prepared using the MAS method with different precursors were evaluated via photodegradation of $\mathrm{RhB}$ dye in a $10 \mathrm{mgL}^{-1}$ aqueous solution under UV light irradiation. There was no obvious photocatalytic degradation of $\mathrm{RhB}$ in the absence of photocatalyst (see Fig. 3(a)). The results showed that approximately $4 \%$ solution discoloration occurred at $210 \mathrm{~min}$ for all materials, and equilibrium was reached at $15 \mathrm{~min}$ of reaction. Thus, in all photocatalytic experiments, the dye solution was kept in the dark for 15 min under stirring. Remarkably, the experiment to verify the photolysis of $\mathrm{RhB}$ in the absence of photocatalyst showed that the RhB hardly decomposed under UV radiation even after 210 min (see Table 2). In contrast, the $\mathrm{ZnS}$ samples present good photocatalytic activities, resulting in a significant reduction in the maximum absorption spectra of RhB (554 nm) during the photodegradation process (see Fig. 3(b-d)). In order to obtain a more appropriate comparison to the well-known and widely applied standards, the synthesized materials were compared with commercial TiO2 anatase (Aldrich, 99.7\%) activity, as shown in Fig. 3-(e-f). These results are consistent with other studies [74-76], and hence they reveal a higher photocatalytic activity for ZnS NPs prepared by the MAS method when compared with anatase $\mathrm{TiO} 2$ crystals. The degradation ratio $\left(\mathrm{C} / \mathrm{C}_{0}\right) \mathrm{RhB}$ solution with $\mathrm{ZnS}$ NPs and without catalyst versus time and the linear relationship of 
[-ln $\left.\left(\mathrm{C} / \mathrm{C}_{0}\right)\right]$ with irradiation time are shown in Fig. 3(e-f).

The photodegradation reaction kinetics of $\mathrm{RhB}$ follow a pseudo-first order rate equation. The values of the rate constant can be obtained from the slope of the $\left[-\ln \left(C_{0} / C_{t}\right)\right]=k_{a p p} t$, where $C_{0}$ and $C_{t}$ are concentrations of $\mathrm{RhB}$ at time zero and $t$, respectively, and $k_{\text {appt }}$ is the apparent first-order rate constant [77]. Correlation coefficients listed in Table $2\left(\mathrm{R}^{2}>0.90\right)$ demonstrated a linear relationship between $\left[-\ln \left(\mathrm{C}_{0} / \mathrm{C}_{\mathrm{t}}\right)\right]$ and reaction time. Thus, the degradation reaction in the RhB solution catalyzed by ZnS NPs occurs according to model pseudo-first kinetic order.

The ZnS NPs synthesized with ZN exhibited a more superior performance than the samples synthesized with $\mathrm{ZC}$ and $\mathrm{ZA}$ (see Table 2): $97 \%$ of the RhB molecules degraded in the presence of $\mathrm{ZnS}$ synthesized with $\mathrm{ZN}$ after $210 \mathrm{~min}$ of exposure to UV radiation, while the samples synthesized with ZC and ZA degraded $82 \%$ and $56 \%$ of the $\mathrm{RhB}$ molecules, respectively. Clearly, the influence of the precursors as surface modifier of these NPs prepared by the MAS method has played a decisive role in the photocatalytic performance enhancement and allows tailored surface chemistry of ZnS NPs. Through the different precursors used it is possible to observed an influence on the exposed surface facet of the ZnS NPs prepared by the MAS method, leading to acquisition of a non-uniform surface composition (confirmed via XPS analysis), and hence the formation and migration rate of the electronhole pairs of such particles as well as the specific surface area of these catalyst affecting the heterogeneous processes. Although an increase occurs in specific surface area of the ZnS NPs governed by different precursors and our results reveal how the reaction rate of the photocatalytic process decreases in this sequence: $\mathrm{ZN}>\mathrm{ZC}>\mathrm{ZA}$. In the photocatalytic experiments, a heterogeneous process takes place on the surface of the photocatalyst and then an analysis of the photocatalytic activity of these materials was obtained using the ratio of the rate constant and the specific surface area of the material, instead of using only the degradation percentage or the reaction constant $(\mathrm{k})$. The relationship between the values of the reaction constant with specific surface area (k/SSA) is shown in Table 2.

Based on the obtained results, we can show that up to $150 \mathrm{~min}$ of reaction the photocatalytic behavior of the both $\mathrm{ZN}$ and $\mathrm{ZC}$ samples is quite similar, and hence are far superior to present by the ZA sample. From the 150 min onward, the photocatalytic activity of the ZC and ZA samples becomes stabilized, whereas for the $\mathrm{ZN}$ sample this stabilization occurs only at $210 \mathrm{~min}$.

The redox ability of $\mathrm{ZnS}$ can be obtained by determining the energy positions of both conduction band (CB) and valence band (VB) edges. Generally, the more positive the potentials of the VB are, the stronger the holes of oxidation capacity are while the more negative the potentials of the $\mathrm{CB}$ are, the stronger the electrons of reduction ability are [78]. According with the band model, in principle, the VB and CB edges positions can be calculated according to the equation as follows [79]:

$E_{V B}=\chi-E^{e}+0.5 E_{g}$

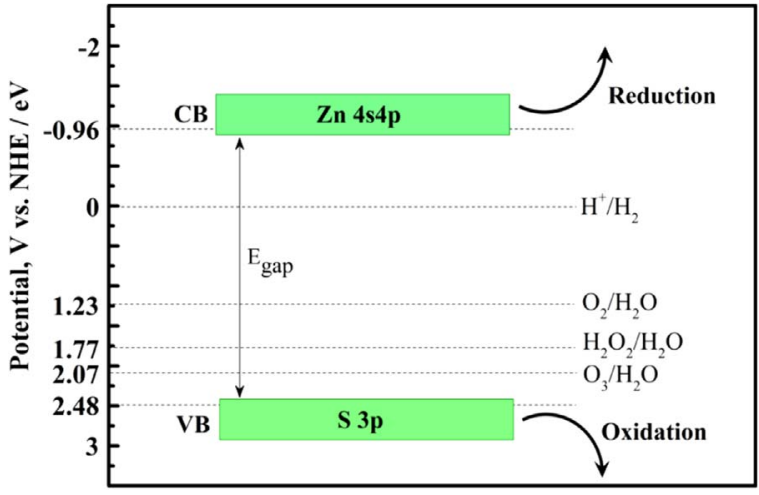

Fig. 4. : Schematic diagram for the redox ability of ZnS NPs.

$E_{C B}=E_{V B}-E_{g}$

where $\mathrm{E}_{\mathrm{VB}}$ and $\mathrm{E}_{\mathrm{CB}}$ are the $\mathrm{VB}$ and $\mathrm{CB}$ edges potentials, respectively. The $\chi$ being the Mulliken electronegativity, which is calculated as the arithmetic mean of the atomic electron affinity and the first ionization energy of the constituent atoms in the semiconductor [80,81]. So in principle, the $\chi$ is calculated to be about $5.26 \mathrm{eV}$ for the $\mathrm{ZnS}[78,81] . E^{e}$ is the energy of free electrons on the hydrogen scale $(\sim 4.5 \mathrm{eV})$ and $E_{g}$ is the band gap energy values. Fig. 4 shows the schematic diagrams (V versus NHE/eV) for ZnS NPs as photocatalyst. In particular, the VB maximum potential for as-prepared $\mathrm{ZnS}$ illustrate a variation in the values from $2.485 \mathrm{eV}$ for $\mathrm{ZN}$ to $2.520 \mathrm{eV}$ for $\mathrm{ZC}$ and $\mathrm{ZA}$, respectively, which in all cases is more positive than $\mathrm{O}_{2} / \mathrm{H}_{2} \mathrm{O}(1.23 \mathrm{~V}), \mathrm{H}_{2} \mathrm{O}_{2} / \mathrm{H}_{2} \mathrm{O}$ $(1.77 \mathrm{~V})$ and $\mathrm{O}_{3} / \mathrm{H}_{2} \mathrm{O}(2.07 \mathrm{~V})$ [82]. Therefore, our results reveals that the $\mathrm{ZnS}$ as-prepared has the ability to oxidize $\mathrm{H}_{2} \mathrm{O}$ to produce $\mathrm{O}_{2}$ and decompose many organic pollutants. The $\mathrm{CB}$ minimum potential of asprepared $\mathrm{ZnS}$ illustrate a variation in the values is about $-0.965 \mathrm{eV}$ for $\mathrm{ZN},-1.020 \mathrm{eV}$ for $\mathrm{ZC}$ and $-1.000 \mathrm{eV}$ for $\mathrm{ZA}$, which is more negative than $\mathrm{H}^{+} / \mathrm{H}_{2}(0 \mathrm{~V})$, and hence can reduce $\mathrm{H}^{+}$to $\mathrm{H}_{2}$, which shows that $\mathrm{ZnS}$ can split water into $\mathrm{H}_{2}$. These results calculated for the top of the $\mathrm{VB}$ and the bottom of the CB for the as-prepared ZnS NPs are in good agreement with literature values [78,81], and hence reveals a high redox ability for such samples.

We propose a model to explain the relevant elementary steps involved in the adsorbed and dissociation process of the $\mathrm{H}_{2} \mathrm{O}$ and $\mathrm{O}_{2}$ species on the $\mathrm{ZnS}$ surface, in accordance with the complex clusters based on the Kröger-Vink notation, and can be summarized by the following equations:

$\left[\mathrm{ZnS}_{4}\right]^{\mathrm{x}}+\left[\mathrm{ZnS}_{3} . \mathrm{V}_{\mathrm{s}}^{\mathrm{x}}\right] \rightarrow\left[\mathrm{ZnS}_{4}\right]^{\prime}+\left[\mathrm{ZnS}_{3} . \mathrm{V}_{\mathrm{s}}^{\bullet}\right]$

$\left[\mathrm{ZnS}_{3} . \mathrm{V}_{\mathrm{s}}^{\bullet}\right]+\mathrm{H}_{2} \mathrm{O} \rightarrow\left[\mathrm{ZnS}_{3} . \mathrm{V}_{\mathrm{s}}^{\bullet}\right] \cdots \mathrm{OH}_{2(\mathrm{ads})}$

$\left[\mathrm{ZnS}_{3} . \mathrm{V}_{\mathrm{s}}^{\bullet}\right] \cdots \mathrm{OH}_{2(\mathrm{ads})} \rightarrow\left[\mathrm{ZnS}_{3} \mathrm{~V}_{\mathrm{s}}^{\mathrm{x}}\right] \cdots \mathrm{OH}^{*}+\mathrm{H}^{\bullet}$

$\left[\mathrm{ZnS}_{3} \cdot \mathrm{V}_{\mathrm{s}}^{\bullet}\right]+\mathrm{O}_{2} \rightarrow \quad\left[\mathrm{ZnS}_{3} \cdot \mathrm{V}_{\mathrm{s}}^{\bullet}\right] \cdots \mathrm{O}_{2(\mathrm{ads})}$

Table 2

Parameter and linear kinetic equation of photocatalytic reaction of the ZnS NPs prepared with different precursors.

\begin{tabular}{|c|c|c|c|c|}
\hline Sample & $\begin{array}{l}\text { Photodecomposition } \\
\text { RhB (\%) } 210 \text { min }\end{array}$ & $\begin{array}{l}\text { Velocity } \\
\text { constant } k \\
\mathbf{x 1 0}^{-3}\left(\min ^{-1}\right)\end{array}$ & $\mathrm{R}^{2}$ & $\begin{array}{l}k / \text { SSA } \\
\left(\min ^{-1} \cdot \mathrm{m}^{-2} \cdot g\right)\end{array}$ \\
\hline $\begin{array}{c}\text { Without } \\
\text { cata- } \\
\text { lysts }\end{array}$ & 12 & 0.54 & 0.9548 & - \\
\hline $\mathbf{Z A}$ & 56 & 4.08 & 0.9796 & 0.0148 \\
\hline $\mathrm{ZC}$ & 82 & 7.96 & 0.9837 & 0.028 \\
\hline $\mathbf{Z N}$ & 97 & 14.05 & 0.9010 & 0.060 \\
\hline $\mathrm{TiO}_{2}$ & 44 & 2.61 & 0.9560 & - \\
\hline
\end{tabular}


$\left[\mathrm{ZnS}_{4}\right]^{\prime}+\left[\mathrm{ZnS}_{3} \cdot \mathrm{V}_{\mathrm{s}}^{*}\right] \cdots \mathrm{O}_{2(\mathrm{ads})} \rightarrow\left[\mathrm{ZnS}_{4}\right]^{\mathrm{x}}+\left[\mathrm{ZnS}_{3} \cdot \mathrm{V}_{\mathrm{s}}^{\bullet}\right] \cdots \mathrm{O}_{2}^{\prime}$

$\left[\mathrm{ZnS}_{3} \cdot \mathrm{V}_{\mathrm{s}}^{\bullet}\right] \cdots \mathrm{O}_{2}^{\prime}+\mathrm{H}^{\bullet} \rightarrow\left[\mathrm{ZnS}_{3} \cdot \mathrm{V}_{\mathrm{s}}^{\bullet}\right] \cdots \mathrm{O}_{2} \mathrm{H}^{*}$

We note that the structural defects on the crystal surface play an important role as the active surface sites responsible for dissociation of adsorbed $\mathrm{H}_{2} \mathrm{O}$ and $\mathrm{O}_{2}$ species and lead to the production of $\mathrm{OH}^{*}$ and $\mathrm{O}_{2} \mathrm{H}^{*}$ radicals, which are the most strongly oxidizing species in this process. Through a combination of scanning tunneling microscopy (STM) experiments and DFT calculations, Schaub et al. [83] reported the unambiguous determination of the active surface sites responsible for dissociation of water molecules adsorbed on the (110) surface of rutile $\mathrm{TiO}_{2}$, i.e., water dissociation was only energetically feasible at the defect sites in the (110) surface of the $\mathrm{TiO}_{2}$. On the other hand, Li et al. [84] presented a systematic study of the facet-dependent photoelectrochemical performance of anatase $\mathrm{TiO}_{2}$ nanomaterials with predominantly exposed (101), (010), and (001) $\mathrm{TiO}_{2}$ surfaces; their results were based on a combination of photocatalysis measurements, characterization of photo-electrochemical performance in dye-sensitized solar cell devices, time-resolved spectroscopy, and first-principles calculations. This suggests that the photochemical performance highly correlates with the surface energy and number of under-coordinated surface atoms, and hence facilitate the formation rate of the $\mathrm{OH}^{*}$ and $\mathrm{O}_{2} \mathrm{H}^{*}$ radicals. In addition, Dong et al. [85] have reported theoretical calculations on the distorted tetrahedral $\left[\mathrm{ZnS}_{4}\right]$ clusters in $\mathrm{WZ} \mathrm{ZnS}$ systems could in principle be beneficial to the separation process of the photogenerated charge carriers. These theoretical results are in very good agreement with our experimental findings.

At this stage, however, it is important to note that direct comparison of theoretical and experimental results is essential and necessary to provide in depth understanding of the electronic and structural properties of NPs and their dependence on the synthetic route and environmental growth conditions. Theoretical calculations can be used not only for interpretation of experiments, but also for prediction of important aspects of new properties that emerge at the nanoscale $[51,86,87]$. The morphology, surface DOS, and surface Fermi level position (and the reliability of the theory to predict these) is key for explaining the physical and chemical behavior at the molecular level, because there is a high surface-to-bulk ratio and these effects are capable of governing the optical, electronic, and photocatalytic properties $[86,87]$. As far as we know, to date, systematic studies towards revealing the surface-structure and facet-dependent catalytic activity relationships of the $\mathrm{ZnS}$ nanocrystals are still very lacking, and hence remain a fascinating challenge to theoretical and experimental researchers. Therefore, theoretical simulations were performed at the DFT level to gain a better understanding of the experimental data, to distinguish between the bulk and surface contributions to the DOS, and to resolve the structural and electronic effects on the physical and chemical properties of the WZ ZnS NPs prepared using the MAS method with different precursors.

Furthermore, it is expected that the specific surface area of ZnS NPs plays a key role during photocatalytic degradation of $\mathrm{RhB}$ dye molecules; therefore, both the surface atomic structure and electronic band structure were systematically investigated via DFT calculations. This has enabled the understanding of the atomic and electronic properties of a crystal surface, which has provided some insight into

Table 3

Number (n) of ZnS units, values of surface area and surface energy $\left(\mathrm{E}_{\mathrm{suf}}\right)$, and the band gap energies ( $\left.E_{g a p}\right)$ for (0001), (1010), (1120) (1012) and (1011) surfaces of ZnS.

\begin{tabular}{lllll}
\hline & $\mathbf{n}$ & $\operatorname{Area~}\left(\mathbf{m}^{\mathbf{2}}\right)$ & $\mathbf{E}_{\mathbf{s u r f}}\left(\mathbf{J} \cdot \mathbf{m}^{-2}\right)$ & $\mathbf{E}_{\boldsymbol{g a p}}(\mathbf{e V})$ \\
\hline $\mathbf{( 0 0 0 1 )}$ & 40 & 51.3 & 1.40 & 1.5 \\
$\mathbf{( 1 0 1 0 )}$ & 12 & 24.3 & 0.56 & 2.1 \\
$(\mathbf{1 1 2 0 )}$ & 24 & 42.0 & 0.41 & 2.3 \\
$\mathbf{( 1 0 1 \overline { 2 } )}$ & 12 & 35.3 & 1.24 & 1.24 \\
$(\mathbf{1 0 1 \overline { 1 } )}$ & 20 & 70.6 & 1.11 & 1.6 \\
\hline
\end{tabular}

the features of single crystal facets relevant to subsequent technological applications [88-90]. Table 3 shows that the calculated surface energy

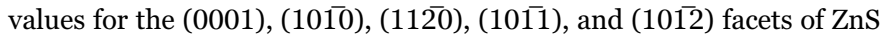
are $1.40,0.56,0.41,1.11$, and $1.24 \mathrm{Jm}^{-2}$, respectively. The (112-0) surface is the most stable facet and the surface stability order agrees with that shown in other theoretical results [52,91]. Different $\mathrm{ZnS}$ surfaces have different catalytic activities, with (0001) being the most reactive surface. An analysis of the results reported in Table 3 reveals a relationship between the energy gap and surface energy. The lower value of the band gap arises from the main surface reactivity of the exposed face during catalytic processes, which provides a valuable tool for interpretation of the experimental results. The results of the analysis of the projected total DOS of the (0001), (1010), (1011), and (1120) facets of $\mathrm{ZnS}$ are shown in Fig. 5(a-d).

Insights from theoretical calculations and experimental evidence can be related to develop a simple model that can be used to better understand crystal facet modulation with the aim of providing new insights into the structural design of the NPs as well as optical and photocatalytic mechanisms at the atomic level. The Wulff construction is a well-recognized method for determining the equilibrium morphology of complex functional materials [43-50,91,92], providing valuable thermodynamic information about frequent crystalline surface energies and constructing the most stable crystal shape.

Fig. 6(a) shows the predicted crystal morphology based on the Wulff construction derived from the calculated surface energy values reported in Table 3. In particular, the Visualization for Electronic and Structural Analysis (VESTA) program (version 3.1.8 for Windows) [93] was used for the Wulff construction as well as in the visualization of the morphological mapping of $\mathrm{ZnS}$ crystals.

Indeed, the TEM results reported in the Supporting Information show that the ZnS samples have an irregular spherical-like shape. Theoretical results predict a cylindrical-like morphology for WZ ZnS with a large non-polar area exposing the (1120) facets and (0001) and (1012) facets at the top and bottom of the cylindrical-like morphology of $\mathrm{ZnS}$ nanocrystals (see Fig. 6(a)), which is in agreement with other reported results [91].

It is important to note that present theoretical results consider the crystals to be in vacuum. The exposed surface planes during the crystal growth process (in particular, those crystal facets with large surface energies) will shrink or even diminish rapidly during the crystal growth process as a result of minimizing the surface energy of small crystals [94], these eventually disappear or have decreased participation in the generated morphology. In this way, the surface is usually encircled by planes with lower surface energies, resulting in a slower crystal growth rate [95]. In this sense, the achieved morphology can be modulated by changing the surface energies of the different exposed surface planes during the crystal growth process thereby modifying the nanomaterial properties.

Basically, controlling the crystal growth process is complex and very sensitive to the chemical environment and internal crystal structures mandated by the synthesis strategy. The type of precursor can be selectively adsorbed onto specific exposed surface planes, thus changing the surface energies and inhibiting a particular active site during the crystal growth process. Therefore, the final morphology based on Wulff's rule will also change. Several insightful review papers have reported experimental and theoretical calculations on the important aspects that govern crystal shape modulation in semiconductor and metal NPs [96-100].

Therefore, in order to obtain the experimental morphology, a proposed hypothesis is that the interaction between precursors decreases the surface energy of the exposed (0001) surface with

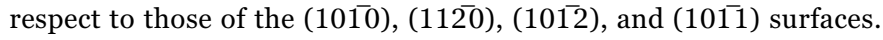
For the present case, a morphological simulation is reported in Fig. 6(b)-(e), in which the Wulff's crystal of the optimized $\mathrm{ZnS}$ and different morphologies are obtained assuming a different surface energy ratio; this generates a controllable and predictable way to 
(a)

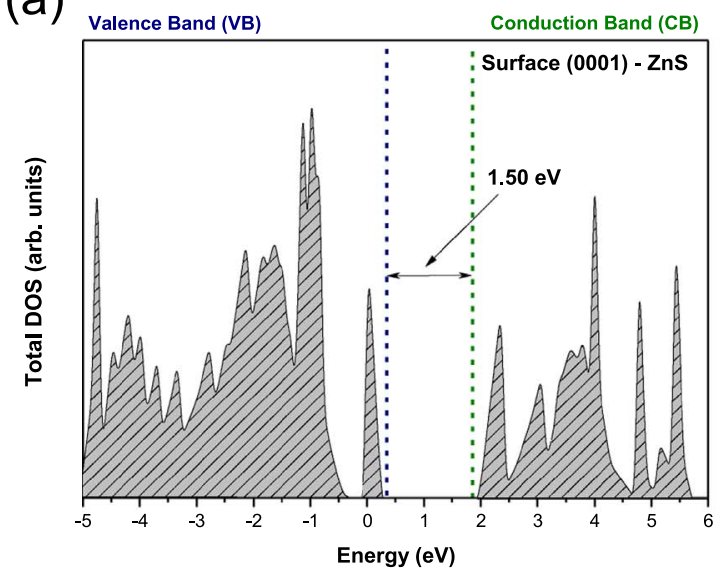

(c)

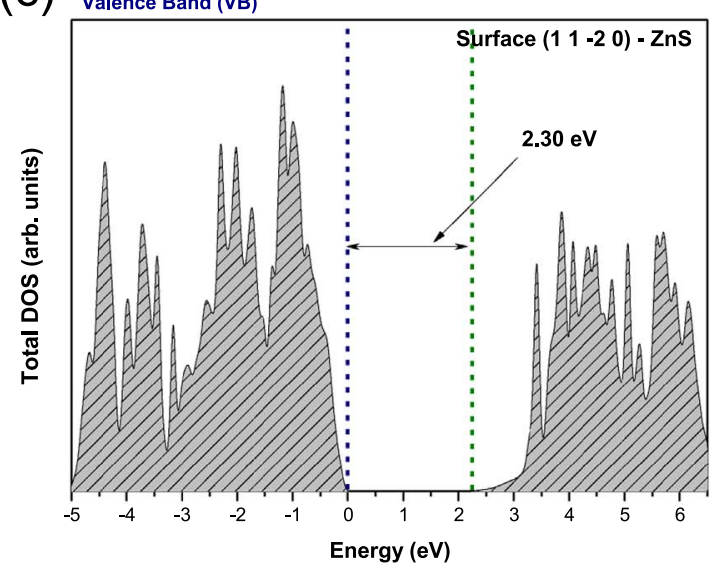

(b)

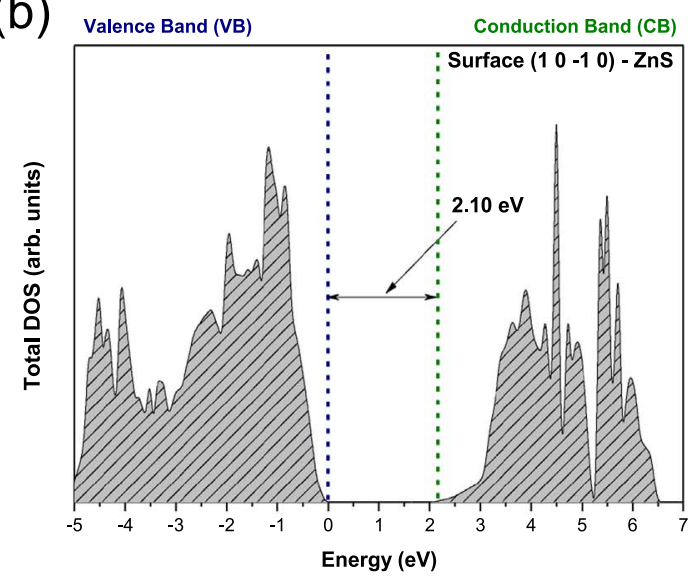

(d)

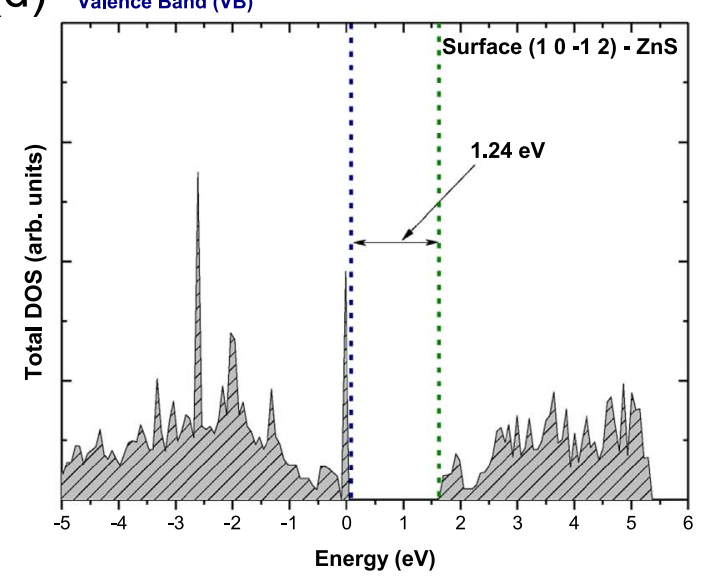

(e) Valence Band (VB)

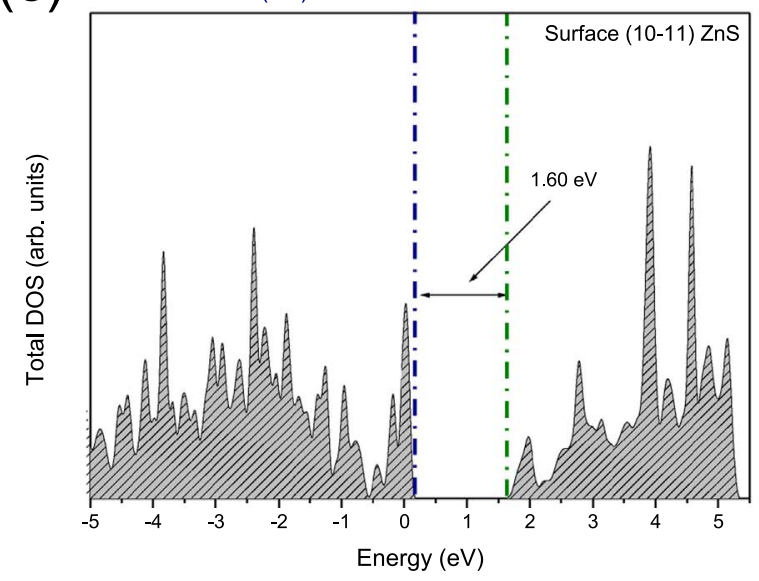

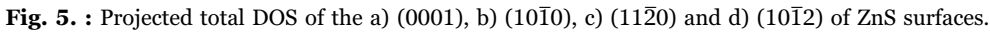

model the morphology in greater depth.

The precursors (i.e., ZN, ZC, or ZA) affect the particle stability and morphology. Theoretically, the achieved morphology depends on the surface energy of each crystal termination. An analysis of Fig. 6 shows that the ideal morphology is a hexagonal prism. When the ratio of the energy surface is varied by decreasing the value of the (0001) surface energy through some perturbation, the results (shown in Fig. 5(b))

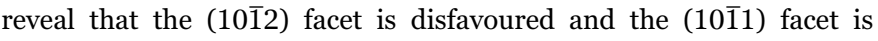
favoured instead. The (1011) surface appears to be favoured with increasing (10 $\overline{1} 2)$ surface energy (shown in Fig. 6(c)). When the ratio of the energy surface is varied by decreasing or increasing the (1010) or $(11 \overline{2} 0)$ surface energy, the (1010) facet is exposed in the morphology (shown in Fig. 6(d)). In particular, a more or less spherical-like

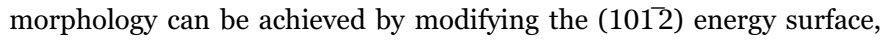
which is in agreement with the experimental TEM images. Therefore, it was found that the experimental morphology can be modulated by tuning the (0001) surface stability, which depends on the precursor.

From this result, it is suggested that the samples synthesized with $\mathrm{ZN}$ present a greater percentage of exposed (0001) surface than the samples synthesized with $\mathrm{ZC}$ and $\mathrm{ZA}$, as revealed by the higher 


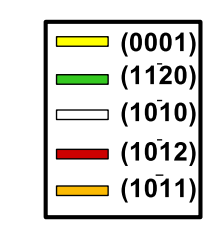

a)

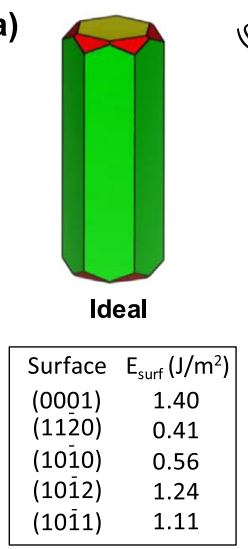

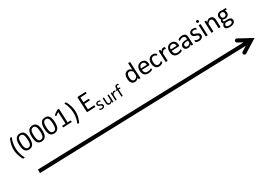

$\stackrel{\text { (10ī2) } \mathrm{E}_{\text {surf }} \text { increasing }}{\longrightarrow}$

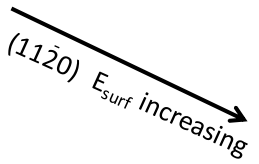

b)

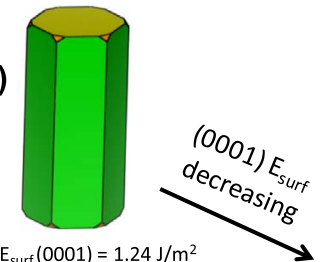

e)

c)
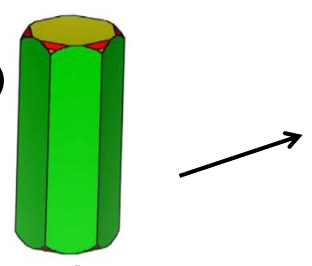

$E_{\text {surf }}(1012)=1.33 \mathrm{~J} / \mathrm{m}^{2}$

d)

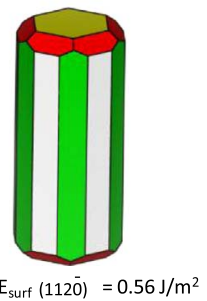

e)

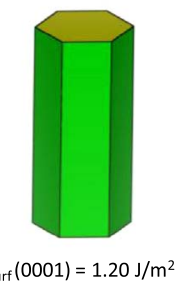

$E_{\text {surf }}(0001)=1.20 \mathrm{~J} / \mathrm{m}^{2}$

Fig. 6. : Morphology map for the ZnS crystal obtained from Wulff's construction and assuming different surface energy values of the different faces.

photocatalytic activity of the former NPs. This fact has important consequences for understanding the photocatalytic mechanism of these NPs, as corroborated by the recently reported study of the facetdependent photocatalytic performance of $\mathrm{ZnO}$ nanostructures [101], which showed the following order for the photodegradation efficiencies of the $(0001)>(101 \overline{1})>(101 \overline{0})$ surfaces $[81,90]$.

This study shows that the different types of defects (i.e., shallow and deep) in ZnS NPs are responsible for generating improved catalytic activity, which is strongly influenced by the nanoscale structural and electronic order-disorder effects. A relationship between the PL behavior, $\rho$, and photocatalytic activity is evident. A close similarity between experimental and theoretical morphologies can be achieved by tuning the (0001) surface stability, which depends on the nature of the precursor as a surface modifier. Therefore, this work is important for a better understanding of the optical, structural, and electronic properties and photocatalytic applications of the ZnS NPs. In this direction, we envision that a more realistic theoretical simulation based on representation of one specific type of surface exposed, combined with a highly sensitive detection of in situ and operando experiments under different conditions, in principle, may provide a unique opportunity to confirm our hypothesis that attempts to describe a close relationship between the photocatalytic activities and PL properties, i.e., as function of the type of its exposed surface and its surface composition (i.e., the acquired understandings of its morphologies) of the as-prepared $\mathrm{ZnS}$ nanocrystals and is a helpful approach to quantitative measurements at the nanoscale.

\section{Conclusions}

In summary, an easy MAS method employing different precursors (i.e., ZN, ZC, or ZA) has been used for the synthesis of ZnS NPs in order to tune optical properties and photocatalytic applications. The results confirm that the hexagonal WZ phase is obtained at low temperatures with a high reaction yield, thereby providing a strategy for obtaining these materials on a large scale. The PL behavior of the ZnS NPs is precursor-dependent and undergoes a red-shift that is linked to specific defects in the crystal framework with maximum emissions at 478, 505, and $555 \mathrm{~nm}$ for the NPs synthesized from $\mathrm{ZN}, \mathrm{ZC}$, and ZA, respectively, when excited by a $350.7 \mathrm{~nm}$ laser line at room temperature. The experimental results demonstrated that the ZnS NPs had good photocatalytic activity for degradation of RhB under UV irradiation, which can be attributed to its unique structure. Increased photocatalytic degradation was observed for samples synthesized using different precursors in the following order: $\mathrm{ZA}<\mathrm{ZC}<\mathrm{ZN}$. Our findings reveal the crucial role of structural defects that can in principle provide a more efficiently separation of the photogenerated charges.

First principles calculations complement experimental work and we have contributed to increasing the knowledge on the structural, chemical and electronic changes of $\mathrm{ZnS}$ and we propose that the samples synthesized with $\mathrm{ZN}$ present a greater percentage of exposed (0001) surface than those synthesized with ZC and ZA. Overall, our investigation clearly reveals that theoretical simulations constitute a helpful strategy to explore experimental conditions and the present study demonstrates that the theoretical findings are in good agreement with the experimental data, and show a connection between the PL behavior and photocatalytic activity of ZnS NPs.

Overall, our investigation clearly reveals that theoretical simulations constitute a helpful strategy to explore experimental conditions. The present synthetic strategy should be a promising fabrication technique for a simple and rapid production of the photocatalysts for the efficient removal of pollutants and open new avenues toward rational design that will benefit the development of novel complex functional materials with desired features and functions. In short, we believe that the focus on factors that control the physical and chemical properties of NPs provides clues that will help contribute to meeting the great demands for environmental sustainability in the near future.

\section{Acknowledgements}

The authors gratefully acknowledge the support from the Brazillian agencies FAPESP (2013/07296-2; 2015/11917-8), CNPq (573636/ 2008-7) and CAPES (process A104/2013). J.A. and L.G acknowledge Prometeo II/2014/022 and ACOMP/2014/270 projects (Generalitat Valenciana), Ministerio de Economia y Competitividad (Spain), CTQ2012-36253-C03-02 and the Spanish Brazilian program (PHB2009-0065-PC, PHBP14/00020) for financially supporting this research. We thank Prof. Dr. P. Hammer (LEFE-IQ/UNESP) for their help with the XPS analysis. We also acknowledge the Servei 
Informática, Universitat Jaume I for the generous allotment of computer time.

\section{Appendix A. Supplementary material}

Supplementary data associated with this article can be found in the online version at doi:10.1016/j.jpcs.2016.12.025.

\section{References}

[1] Z. Zhang, J. She, H. Chen, S. Deng, N. Xu, J. Mater. Chem. C 1 (2013) 4970-4978.

[2] X. Fang, T. Zhai, U.K. Gautam, L. Li, L. Wu, Y. Bando, D. Golberg, Prog. Mater. Sci. 56 (2011) 175-287.

[3] S. Shen, Q. Wang, Chem. Mater. 25 (2013) 1166-1178.

[4] F.A. La Porta, J. Andrés, M.S. Li, J.R. Sambrano, J.A. Varela, E. Longo, Phys. Chem. Chem. Phys. 16 (2014) 20127-20137.

[5] C.S. Tiwary, S. Saha, P. Kumbhakar, K. Chattopadhyay, Cryst. Growth Des. 14 (2014) 4240-4246.

[6] B. Liu, Q. Liu, C. Tong, Colloids Surf. A 434 (2013) 213-219.

[7] Y. Zhu, Q. Ruan, F. Xu, Nano Res. 2 (2009) 688-694.

[8] A. Kumar, R.K. Upadhyay, J. Mater. Sci: Mater. Electron. 26 (2015) 2430-2435.

[9] F.A. La Porta, M.M. Ferrer, Y.V.B. Santana, C.W. Raubach, V.M. Longo, J.R. Sambrano, E. Longo, J. Andrés, M.S. Li, J.A. Varela, J. Alloy. Compd. 556 (2013) 153-159.

[10] N. Srinivasan, S. Thirumaran, C. R. Chimie., 17, 2014, pp. 964-970.

[11] H. Zhang, B.R. Hyun, F.W. Wise, R.D. Robinson, Nano Lett. 12 (2012) $5856-5860$.

[12] Z.Q. Mamiyev, N.O. Balayeva, Chem. Phys. Lett. 646 (2016) 69-74.

[13] S. Ummartyotin, Y. Infahsaeng, Renew. Sustain. Energy Rev. 55 (2016) 17-24.

[14] N.H. Abdullah, Z. Zainal, S. Silong, M.I.M. Tahir, K.B. Tan, S.K. Chang, Mater. Chem. Phys. 176 (2016) 33-41.

[15] K. Deka, M.P.C. Kalita, Braz. J. Phys. 45 (2015) 36-40.

[16] L. Yin, D. Zhang, D. Wang, X. Kong, J. Huang, F. Wang, Y. Wu, Mater. Sci. Eng. B 208 (2016) 15-21.

[17] P. Hu, G. Gong, F. Zhan, Y. Zhang, R. Li, Y. Cao, Dalton Trans. 45 (2016) $2409-2416$.

[18] R. Ramachandran, M. Saranya, P. Kollu, B.P.C. Raghupathy, S.K. Jeong, A.N. Grace, Eletrochim. Acta 178 (2015) 647-657.

[19] P. Hu, G. Gong, F. Zhan, Y. Zhang, R. Li, Y. Cao, Dalton Trans. 45 (2016) 2409-2416.

[20] K. Vijaianand, M. Karlchinnu, R. Mohankumar, R. Mohan, R. Jayavel, Appl. Surf. Sci. 255 (2009) 8879-8882

[21] X. Yan, E. Michael, S. Komarnenic, J.R. Brownsond, Z.F. Yana, Ceram. Int. 39 (2013) 4757-4763.

[22] L. Wang, P. Wang, B. Huang, X. Ma, G. Wang, Y. Dai, X. Zhang, X. Qin, Appl. Surf. Sci. (2016). http://dx.doi.org/10.1016/j.apsusc.2016.06.159).

[23] R. Wang, H. Liang, J. Hong, Z. Wang, J. Photochem. Photobiol. A 325 (2016) $62-67$.

[24] M.M. Ferrer, Y.V.B. de Santana, C.W. Raubach, F.A. La Porta, A.F. Gouveia, E. Longo, J.R. Sambrano, J. Mol. Model. 20 (2014) 2375-2383.

[25] Y.V.B. Santana, C.W. Raubach, M.M. Ferrer, F.A. La Porta, J.R. Sambrano, V.M. Longo, E.R. Leite, E. Longo, J. Appl. Phys. 110 (2011) 123507.

[26] L.L. Wang, C.Z. Zhao, L.P. Kang, D.W. Liu, H.C. Zhao, S.P. Hao, Y.K. Zhang, Z.P. Chen, X.J. Li, Appl. Surf. Sci. 384 (2016) 530-533.

[27] S. Kaur, S. Sharma, S.K. Kansal, Superlattices Microstruct. 98 (2016) 86-95.

[28] S. Ahluwalia, N.T. Prakash, R. Prakash, B. Pal, Chem. Eng. J. 306 (2016) 1041-1048.

[29] K. Kalpana, V. Selvaraj, J. Ind. Eng. Chem. 41 (2016) 105-113.

[30] R. Bose, U. Thupakula, J.K. Bal, N. Pradhan, J. Phys. Chem. C. 116 (2012) $16680-16686$.

[31] J.G. Zhao, H.H. Zhang, Superlattices Microstruct. 51 (2012) 663-667.

[32] S. Li, Z. Wu, W. Li, Y. Lin, R. Zhuo, D. Yan, W. Jun, P. Yan, CrystEngComm 15 (2013) 1571-1577.

[33] M.S. Niasari, F. Davar, H. Seyghalkar, E. Esmaeili, N. Mir, CrystEngComm 13 (2014) 2948-2954.

[34] M. Scocioreanu, L. Mihut, M. Baibarac, I. Baltog, Phys. Status Solidi B 250 (2013) 1426-1431.

[35] Y. Cheng, Z. Lin, H. Lu, L. Zhang, B. Yang, Nanotechnology 25 (2014) 115601.

[36] F. Kurnia, Y. Hau, Ng, Y. Tang, R. Amal, N. Valanoor, J.N. Hart, Cryst. Growth Des. 16 (2016) 2461-2465.

[37] C.W. Raubach, Y.V.B. de Santana, M.M. Ferrer, P.G.C. Buzolin, J.R. Sambrano, E. Longo, Dalton Trans. 42 (2013) 11111-11116.

[38] S. Xiong, B. Xi, C. Wang, D. Xu, X. Feng, Z. Zhu, Y. Qian, Adv. Funct. Mater. 17 (2007) 2728-2738.

[39] X. Yu, J. Yu, B. Cheng, B. Huang, Chem. Eur. J. 15 (2009) 6731-6739.

[40] H.S. Park, W.B. Ko, J. Nanosci. Nanotechnol. 14 (2014) 8646-8653.

[41] H. Naeimi, H. Foroughi, New J. Chem. 39 (2015) 1228-1236.

[42] S. Sarkar, A.K. Guria, N. Pradhan, Chem. Commun. 49 (2013) 6018-6020.

[43] M.R.D. Bomio, R.L. Tranquilin, F.V. Motta, C.A. Paskocimas, R.M. Nascimento, L. Gracia, J. Andres, E. Longo, J. Phys. Chem. C. 117 (2013) 21382-21395.

[44] R.A. Roca, J.C. Sczancoski, I.C. Nogueira, M.T. Fabbro, H.C. Alves, L. Gracia, L.P.S. Santos, C.P. de Sousa, J. Andrés, G.E. Luz Jr, E. Longo, L.S. Cavalcante, Catal. Sci. Technol. 5 (2015) 4091-4107.
[45] R.A. Roca, P.S. Lemos, J. Andrés, E. Longo, Chem. Phys. Lett. 644 (2016) 68-72.

[46] M.T. Fabbro, C. Saliby, L.R. Rios, F. La Porta, L. Gracia, M. Li, J. Andrés, L.P. Serejo dos Santos, E. Longo, Sci. Technol. Adv. Mater. 16 (2015) 065002.

[47] R.C. de Oliveira, M. Assis, M.M. Teixeira, M.D.P. da Silva, M.S. Li, J. Andres, L. Gracia, E. Longo, J. Phys. Chem. C. 120 (2016) 12254-12264.

[48] M.T. Fabbro, L. Gracia, G.S. Silva, L.P.S. Santos, J. Andrés, E. Cordoncillo, E. Longo, J. Solid State Chem. 239 (2016) 220-227.

[49] G. Botelho, J. Andres, L. Gracia, L.S. Matos, E. Longo, Chem. PhysChem. 81 (2016) 202-212.

[50] G. Botelho, J.C. Sczancoski, J. Andrés, L. Gracia, E. Longo, J. Phys. Chem. C. 119 (2015) 6293-6306.

[51] F.A. La Porta, L. Gracia, J. Andrés, J.R. Sambrano, J.A. Varela, E. Longo, J. Am. Ceram. Soc. 97 (2014) 4011-4018.

[52] C.A. Feigl, A.S. Barnard, S.P. Russo, Phys. Chem. Chem. Phys. 14 (2012) 9871-9879.

[53] X. Meng, H. Xiao, X. Wen, W. Goddard III, A., S. Li, G. Qin, Phys. Chem. Chem. Phys. 15 (2013) 9531-9539.

[54] E. Balantseva, G. Berlier, B. Camino, M. Lessio, A.M. Ferrari, Study, J. Phys. Chem. C 118 (2014) 23853-23862.

[55] J.M. Azpiroz, E. Mosconi, F.D. Angelis, J. Phys. Chem. C 115 (2011) $25219-25226$.

[56] J.M. Azpiroz, E. Mosconi, J.M. Ugalde, F.D. Angelis, J. Phys. Chem. C 118 (2014) 3274-3284

[57] H. Zhang, B. Gilbert, F. Hung, J.F. Banfield, Nature 424 (2003) 1025-1029.

[58] G. Kresse, G.J. Hafner, Phys. Rev. B 49 (1994) 14251.

[59] G. Kresse, G.J. Furthmuller, Comput. Mater. Sci. 6 (1996) 15-50.

[60] J.P. Perdew, K. Burke, M. Ernzerhof, Phys. Rev. Lett. 77 (1997) 3865.

[61] G. Kresse, D. Joubert, Phys. Rev. B 59 (1999) 1758.

[62] H.J. Monkhorst, J.D. Pack, Phys. Rev. B: Solid State 13 (1976) 5188.

[63] J.F. Xu, W. Ji, J.Y. Lin, S.H. Tang, Y.W. Du, Appl. Phys. A 66 (1998) 639-641.

[64] C.D.Wagner, G.E.Muilenberg, Handbook of X-ray photoelectron spectroscopy: a reference book of standard data for use in x-ray photoelectron spectroscopy, Physical Electronics Division, Perkin-Elmer Corp, 1979.

[65] Y. -P. Zhu, J. Li, T.-Y. Ma, Y. -P. Liu, G. Du, Z. -Y. Yuan, J. Mater. Chem. A 2 (2014) 1093-1101.

[66] V. Etacheri, M.K. Seery, S.J. Hinder, S.C. Pillai, Adv. Funct. Mater. 21 (2011) 3744-3752.

[67] V. Kumar, V. Kumar, S. Som, J.H. Neethling, M. Lee, O.M. Ntwaeaborwa, H.C. Swart, Nanotechnology 25 (2014) 135701-135709.

[68] J. -S. Hu, L. -L. Ren, Y. Guo, H. -P. Liang, A. -M. Cao, L. -J. Wan, C. -L. Bai, Angew. Chem. 117 (2005) 1295-1299.

[69] F.A. La Porta, J. Andrés, M.V.G. Vismara, C.F.O. Graeff, J.R. Sambrano, M.S. Li, J.A. Varela, E. Longo, J. Mater. Chem. C 2 (2014) 10164-10174.

[70] J.M. Ziman, Models of Disorder: the Theoretical Physics of Homogeneously Disordered Systems, Cambridge University Press, Cambridge, 1979, p. 525.

[71] S.K. Estreicher, T.M. Gibbons, M.B. Bebek, J. Appl. Phys. 117 (2015) 112801.

[72] Y. Gong, T. Andelman, G.F. Neumark, S. O’Brien, I.L. Kukovsky, Nanoscale Res. Lett. 2 (2007) 297-302.

[73] E. Rauwel, A. Galeckas, P. Rauwel, M.F. Sunding, H. Fjellvag, J. Phys. Chem. C 115 (2011) 25227-25233.

[74] U.G. Akpan, B.H. Hameed, J. Hazard. Mater. 170 (2009) 520.

[75] G. Plantard, T. Janin, V. Goetz, S. Brosillon, Appl. Catal. B 115-116 (2012) 38-44.

[76] D. Yang, H. Liu, Z. Zheng, Y. Yuan, J.-C. Zhao, E.R. Waclawik, X. Ke, H. Zhu, J. Am. Chem. Soc. 131 (2009) 17885-17893.

[77] M. Huang, S. Weng, B. Wang, J. Hu, X. Fu, P. Liu, J. Phys. Chem. C 118 (2014) 25434-25440.

[78] X. Chen, S. Shen, L. Guo, S.S. Mao, Chem. Rev. 110 (2010) 6503-6570.

[79] Y. Xu, M.A.A. Schoonen, Am. Mineral. 85 (2000) 543-556.

[80] H. Shi, Z. Li, J. Kou, J. Ye, Z. Zou, J. Phys. Chem. C 115 (2010) 145-151.

[81] C. Mondal, A. Singh, R. Sahoo, A.K. Sasmal, Y. Negishi, T. Pal, New J. Chem. 39 (2015) 5628-5635.

[82] J. Tang, J. Ye, Chem. Phys. Lett. 410 (2005) 104.

[83] R. Schaub, P. Thostrup, N. Lopez, E. Laegsgaard, I. Stensgaard, J.K. Nørskov, F. Besenbacher, Phys. Rev. Lett. 87 (2001) 266104.

[84] C. Li, C. Koenigsmann, W. Ding, B. Rudshteyn, K.R. Yang, K.P. Regan, S.J. Konezny, V.S. Batista, G.W. Brudvig, C.A. Schmuttenmaer, J. -H. Kim, J. Am. Chem. Soc. 137 (2015) 1520-1529.

[85] M. Dong, J. Zhang, J. Yu, APL Mater. 3 (2015) 104404.

[86] J.K. Nørskov, T. Bligaard, J. Rossmeisl, C.H. Christensen, Nat. Chem. 1 (2009) $37-46$.

[87] M. Hjort, S. Lehmann, J. Knutsson, A.A. Zakharov, Y.A. Du, S. Sakong, R. Timm, G. Nylund, E. Lundgren, P. Kratzer, K.A. Dick, A. Mikkelsen, ACS Nano 8 (2014) $12346-12355$.

[88] J. Andrés, L. Gracia, A.F. Gouveia, M.M. Ferrer, E. Longo, Nanotechnology 26 (2015) 405703.

[89] M.M. Ferrer, A.F. Gouveia, L. Gracia, E. Longo, J. Andrés, Model. Simul. Mater. Sci. Eng. 24 (2016) 025007.

[90] M.C. Oliveira, L. Gracia, I.C. Nogueira, M.F.C. Gurgel, J.M.R. Mercury, E. Longo, J. Andrés, Ceram. Inter. 42 (2016) 10913.

[91] S. Hamad, S. Cristol, C.R.A. Catlow, J. Phys. Chem. B 106 (2002) 11002-11008.

[92] A.S. Barnard, C.A. Feigland, S.P. Russo, Nanoscale 2 (2010) 2294-2301.

[93] K. Momma, F. Izumi, J. Appl. Crystallogr. 44 (2011) 1272.

[94] C. -F. Huo, B. -S. Wu, P. Gao, Y. Yang, Y. -W. Li, H. Jiao, Angew. Chem. 123 (2011) 7541-7544.

[95] C. Burda, X.B. Chen, R. Narayanan, M.A. El-Sayed, Chem. Rev. 105 (2005) 1025-1102. 
[96] M.A. Lovette, A.R. Browning, D.W. Griffin, J.P. Sizemore, R.C. Snyder, M.F. Doherty, Ind. Eng. Chem. Res. 47 (2008) 9812-9833.

97] A. Seyed-Razavi, I.K. Snook, A.S. Barnard, J. Mater. Chem. 20 (2010) 416-421.

[98] J.Y. Chen, B. Lim, E.P. Lee, Y.N. Xia, Nano Today 4 (2009) 81.

[99] Z.Y. Jiang, Q. Kuang, Z.X. Xie, L.S. Zheng, Adv. Funct. Mater. 20 (2010)
3634-3645.

[100] K. Lee, M. Kim, H. Kim, J. Mater. Chem. 20 (2010) 3791-3798.

[101] Y.V. Kaneti, Z. Zhang, J. Yue, Q.M.D. Zakaria, C. Chen, X. Jiang, A. Yu, Phys. Chem. Chem. Phys. 16 (2014) 11471-11480. 